\title{
Alkaloide der Pareirawurzel
}

von

\author{
Franz Faltis.
}

A:as dem I. chemischen Laboratorium der k. k. Universität Wien.

(Vorgelegt in der Sitzung am 23. Mai 1912.)

Die Alkaloide der Pareirawurzel wurden in der letzten Zeit von $\mathrm{Scholtz}{ }^{1}$ bearbeitet. In seiner ersten Abhandlung findet man eine Übersicht über die früheren Arbeiten auf diesem Gebiete, die bis ins Jahr 1840 zurückreichen. Die Pareirawurzel stammt von einer südamerikanischen Menispermacee, nämlich Chondodendron tomentosum, also aus einer Pflanzenfamilie, für deren Alkaloide ich die Ableitung von einer mehreren verwandten Familien gemeinsamen Muttersubstanz annehme." Scholtz stellte für das Hauptalkaloid, das Bebirin, die Formel $\mathrm{C}_{18} \mathrm{H}_{21} \mathrm{NO}_{3}$ auf, so daß, da die Anwesenheit einer Hydroxyl-, einer Methoxyl- und einer Methylimidgruppe sichergestellt war, für das Gerüst des Moleküls $\mathrm{C}_{16} \mathrm{H}_{14} \mathrm{O}\left(\mathrm{NCH}_{3}\right)(\mathrm{OH})\left(\mathrm{OCH}_{3}\right)$ eine sehr nahe Beziehung zur abgebauten Stammsubstanz, wie sie dem Laudanosin $\mathrm{C}_{16} \mathrm{H}_{12}\left(\mathrm{NCH}_{3}\right)\left(\mathrm{OCH}_{3}\right)_{4}$ zugrunde liegt, anzunehmen war. Unter diesen Umständen entschloß ich mich, die Konstitution des Bebirins näher zu untersuchen, um die Brauchbarkeit meiner oben erwähnten Annahme als Arbeitshypothese auf dem Gebiete der Alkaloidchemie der betreffenden Pflanzengruppe zu prüfen. Doch nötigen mich die Ergebnisse, die ich bei der Analyse des Hauptalkaloids erhielt, demselben die

1 Arch. für Pharm., 236, 530 bis 541 (1898); 237, 199 (1899); 244, 555 bis 560 (1906); 249,408 bis 418 (1911).

2 Pharm. Post, 1906, Nr. 31, 32; 1911, Nr. 52. 
Formel $\mathrm{C}_{21} \mathrm{H}_{23} \mathrm{NO}_{4}=\mathrm{C}_{19} \mathrm{H}_{16} \mathrm{O}_{2}\left(\mathrm{NCH}_{3}\right)(\mathrm{OH})\left(\mathrm{OCH}_{3}\right)$ zu geben, so daß die erwartete einfache Beziehung zum Laudanosin nicht rorhanden ist.

Die Untersuchung der Basen der Pareirawurzel wurde an dem käuflichen Bebirinum sulfuricum von $M$ e r ck vorgenommen, welches nach Angabe der Firma durch Extraktion der Pareirawurzel mit verdünnter Schwefelsäure gewonnen wird und, wie Scholt $z$ angibt, mit dem Alkaloidgemenge übereinstimmt, das er selbst auf diese Weise aus der Wurzel gewonnen hatte.

Ich konnte bis jetzt drei verschiedene Alkaloide aus dem Gemenge der Basen isolieren, das durch Fällen mit $\mathrm{Na}_{2} \mathrm{CO}_{3}$ aus der Sulfatlösung gewonnen wurde. Das Hauptalkaloid erhielt ich ursprünglich wie Scholtz durch Ausziehen mit Äther im Soxhlet, später aber urch das bedeutend rascher vor sich gehende erschöpfende Auskochen mit Benzol, in weichem es allein leicht löslich ist. Es stimmt ganz mit den Angaben der alten Autoren überein, besonders was seinen Mangel an Krystallisierbarkeit als Base sowohl als in Salzform betrifft, zeigte aber zu meiner großen Überraschung nicht die Eigenschaft, die Scholt $z$ als charakteristisch für das Bebirin anführt, nämlich die Fähigkeit, nach dem Lösen in Methylalkohol sich als schwer lösliche krystallinische Form auszuscheiden. Die Analyse der durch öfteres Auflösen in Benzol und fraktioniertes Fällen mit Petroläther gereinigten Base führte, wie bereits erwähnt, zu der abweichenden Formel $\mathrm{C}_{21} \mathrm{H}_{23} \mathrm{NO}_{4}$, so daß ich zuerst meinte, ein von dem Scholtz'schen Bebirin gänzlich verschiedenes Alkaloid in Händen zu haben, obwohl es sehr auffallend war, jenes bei der gleichen Darstellungsweise aus demselben Ausgangsmaterial überhaupt nicht auffinden zu können. Die Verschiedenheit im optischen Drehvermögen beider Basen, das beim Scholtz'schen Bebirin $[\alpha]_{D}^{28}= \pm 298$, bei meinem Alkaloid $[a]_{D}^{21}=+28 \cdot 6$ (in Alkohol) beträgt, legte daher den Gedanken nahe, die Erklärung der Verschiedenheit beider Basen in Stereoisomerie zu suchen, wodurch bei leicht eintretender teilweiser Inaktivierung die Auffindung von verschiedenen Isomeren je nach den Versuchsbedingungen bei der Gewinnung des Extrakts (höhere Temperatur, konzentriertere Säure) und des Salzgemenges verständlich ist. 
Dies hatte natürlich zur Voraussetzung, daß auch dem Bebirin von Scholtz die Formel $\mathrm{C}_{21} \mathrm{H}_{23} \mathrm{NO}_{4}$ zukommt. In der Tat ergaben dies die Verbrennungen, die ich an einem Originalpräparat von Scholtz, das sich seit zirka 14 Jahren im hiesigen Institut befand und alle von Scholtz angegebenen Eigenschaften des krystallinischen Bebirins zeigte, nach dem Umkrystallisieren aus Methylalkohol ausführte. Ein kleiner Teil des Präparates $(0.1 \mathrm{~g})$, der aus Methylakohol nicht auskrystallisierte und durch Eindampfen gewonnen wurde, hatte eine Umwandlung erfahren: es krystallisierte aus Methylalkohol, in dem es leicht löslich war, nicht mehr aus und löste sich nur zur Hälfte in Benzol. Mit Petroläther daraus gefällt, zeigte die Probe einen Schmelzpunkt von $160^{\circ}$ und gab mit meinem Bebirin vermischt eine Schmelzpunktsdepression auf $120^{\circ}$. Dies zeigt die Umwandelbarkeit des Bebirins. Ein zweites Präparat von Scholtz, das sein amorphes Bebirin (aus Methylalkohol in das krystallisierte umwandelbar) enthalten hatte, hat sich in dieser Zeit vollständig verändert: der größte Teil war in Benzol und auch in Chloroform unlöslich. Dieser Anteil hatte einen Schmelzpunkt von $285^{\circ}$ bei raschem Erhitzen, dürfte also eine ähnliche Umwandlung erfahren haben, wie sie mein Bebirin beim längeren Liegen zum kleinen Teil zeigt (siehe später). Dafür spricht auch die Bestimmung der Hydroxylgruppen nach Zerewitin off.

Nach diesem Verhalten des Bebirins ist wohl anzunehmen, daß die alten Autoren, die übereinstimmend die Unfähigkeit des Bebirins, aus irgendeinem Lösungsmittel zu krystallisieren, betonen, wahrscheinlich dieselbe Modifikation in Händen gehabt haben wie ich. Die Folgerung Scholtz', ${ }^{1}$ daß Buxin, das Alkaloid von Buxus sempervirens L., von Bebirin verschieden sein müsse, da es aus Methylalkohol nicht in den krystallisierten Zustand übergeht, ist nach dem Gesagten noch bis auf weitere Untersuchungen als nicht bewiesen zu erachten.

\section{$\beta$-Bebirin.}

Ich lege meinem amorphen, schwach drehenden Alkaloid den Namen $\beta$-Bebirin bei zum Unterschied vom stark drehenden

1 Arcl. für Pharm., 236, 540 (1898). 
x-Bebirin Scholtz', das in zwei Formen, einer amorphen und einer krystallisierten, auftritt. Das $\beta$-Bebirin, das einzige in Benzol leicht lösliche Alkaloid der Pareirawurzel, bildet mit $20 \%$ den Hauptbestandteil des Basengemisches. Es ist in allen organischen Lösungsmitteln außer Petroläther leicht löslich, in Wasser unlöslich. Aus der sauren Lösung wird es durch Soda vollständig gefällt. Sein Schmelzpunkt ist, da es vollständì amorph ist, sehr unscharf. Er liegt bei zirka 142 bis $150^{\circ}$. Sein Drehvermögen beträgt in einer absolut alkoholischen $1 \cdot 64$ prozentigen Lösung, wie schon gesagt, $[\alpha]_{D}^{21}=+28 \cdot 6$, in einer $1 \cdot 7$ prozentigen Pyridinlösung dagegen $[\alpha]_{D}^{21}=-24 \cdot 7$. Die Ursache dieser Umkehrung des Drehvermögens ist wahrscheinlich darin zu suchen, daß Bebirin als Phenol mit Pyridin in der Lösung ein Salz gibt. Das $\beta$-Bebirin gibt keine charakteristischen Farbenreaktionen.

\section{Alkaloid $B$.}

Das zweite Alkaloid, das einstweilen als Base $B$ bezeichnet werde, wurde neben dem dritten durch Auskochen des nach dem Extrahieren mit Benzol zurückbleibenden Basengemisches mit Alkohol als der in Alkohol löslichere Anteil in einem Ausmaße von $7 \%$ gewonnen. Es ist in Pyridin schon in der Kälte sowie in $\mathrm{CHCl}_{3}$ leicht löslich, der Schmelzpunkt (unscharf unter Zersetzung) liegt bei raschem Erhitzen bei $220^{\circ}$. Die Base löst sich in $\mathrm{KOH}$ leicht auf; auch in siedendem Wasser geht sie beträchtlich in Lösung, aus der sauren Lösung wird sie von Soda nicht vollständig gefällt (in beiden Fällen tritt im Filtrat mit $\mathrm{J}_{2}$.JK-Lösung reichliche Fällung ein). Die Analysen ergaben die Formel $\mathrm{C}_{22} \mathrm{H}_{23} \mathrm{NO}_{5}$. Die Base $B$ enthält eine Methoxyl- und eine Methylimidgruppe. Die Bestimmung der Anzahl der Hydroxyle nach Zerewitinoff machte das Vorhandensein von zwei Hydroxylgruppen wahrscheinlich. Die Base dreht den polarisierten Lichtstrahl nach rechts. Die $1 \cdot 07$ prozentige Lösung in Pyridin ergab eine Drehung von $[x] j=+56 \cdot 7$ (allerdings war die Lösung sehr dunkel und mußte mit Auerlicht gearbeitet werden).

Bei der Kalischmelze wurde Protocatechusäure als Spaltprodukt erhalten. 


\section{Isobebirin.}

Das dritte Alkaloid, das in einem Ausmaße von $3 \%$ erhalten wurde, ist in reinem Zustande in Alkohol wie in den meisten organischen Lösungsmitteln sehr schwer, in heißem $\mathrm{CHCl}_{3}$ sowie in kaltem Pyridin ziemlich leicht löslich. Es krystallisiert aus einer mit Methylalkohol versetzten Pyridinlösung beim Eindampfen in rhombischen Nadeln und schmilzt bei raschem Erhitzen bei $290^{\circ}$ unter Zersetzung. Die Base zeigt Phenolcharakter, da sie in $\mathrm{KOH}$ leicht löslich ist, und enthält so wie die anderen beiden Alkaloide eine Methoxyl- und eine Methylimidgruppe. Die Bestimmung nach $Z$ erewitinoff gab Werte, die annähernd auf zwei Hydroxyle stimmen, die Untersuchung des optischen Drehvermögens ergab optische Inaktivität, alllerdings in einer dunkel gefärbten Lösung mit Hilfe der Auerflamme, so daß das Resultat nicht sehr verläßlich ist. Mit Eisenchlorid gibt die wässerige neutrale Lösung des Chlorids eine nach einiger Zeit auftretende, lang andauernde trübolivengrüne Färbung.

Diese Eisenchloridfärbung, die vielleicht auf das Vorhandensein einer freien Brenzcatechingruppe hinweist, hat die Base mit dem Alkaloid gemeinsam, das ich aus dem Bebirinum sulfuricum crystallisatum von $\mathrm{M} \mathrm{erck}$ dargestellt habe. Dieses zeigt in einer $1 \cdot 73$ prozentigen Pyridinlösung das Drehvermögen $[\alpha]_{D}^{22}=-47 \cdot 7$. Ich halte beide für stereoisomer, und zwar das inaktive Alkaloid für das Racemprodukt (falls die Bestimmung des Drehvermögens der inaktiven Substanz verläßlich ist), das sich beim weiteren Eindampfen des Sulfatauszuges aus der aktiven Base durch Selbstracemisierung gebildet hat. ${ }^{1}$

1 Die Verbrennungswerte der inaktiven Base gaben zwar um $0 \cdot 7 \%$ kleinere Werte für Kohlenstoff als das aktive Alkaloid, so daB ich früher (siehe Anzeiger d. kais. Akad. d. Wiss. in Wien, Nr. IX) die Formel $\mathrm{C}_{20} \mathrm{H}_{21} \mathrm{NO}_{4}$ berechnete, die ich auch zuerst durch eine Analyse der noch nicht genügend gereinigten aktiven Base bestätigt fand. Die Substanz hinterließ aber jedesmal nach der Verbrennung einen Rückstand, der jedenfalls von der $\mathrm{Na}_{2} \mathrm{CO}_{3}$-Fällung herrihrte. Die inaktive Base wurde nämlich nie durch Auskrystallisieren, sondern durch Eindampten der Pyridinlösung gewonnen. Der Rückstund wurde einmal gewogen und betrug $0.0020 \mathrm{~g}$. Wird er als $\mathrm{Na}_{2} \mathrm{CO}_{3}$ in Rechnung gezogen, so kommt man 'zu denselben Werten wie bei der aktiven Base (siehe experim. Teil). 
Das Bebirinum sulfuricum crystallisatum wird nämlich nach Angaben, die ich von der Firma Merck erhielt, dadurch gewonnen, daß diese Krystalle beim Einengen des schwefelsauren Auszuges der Pareirawurzel zuerst anschießen, während in der Mutterlauge das Bebirinum sulfuricum amorphum bleibt. Da kein Bedarf nach dem krystallisierten Produkt vorhanden ist, wird im allgemeinen ohne Rücksicht auf dieses alles auf das amorphe Bebirinum sulfuricum verarbeitet. So würde sich das Vorfinden desselben Alkaloids in beiden Präparaten erklären. Aus dem krystallinischen Sulfat erhielt ich das Alkaloid, indem ich die wässerige Lösung mit einem kleinen Überschuß von Sodalösung versetzte und den ausfallenden, fein verteilten rötlichweißen Niederschlag mit $\mathrm{CHCl}_{3}$ ausschüttelte. Aus $5 \mathrm{~g}$ Sulfat wurden $2 g$ reine Base gewonnen. Der Schmelzpunkt derselben liegt bei $295^{\circ}$ bei raschem Erhitzen, die wohlausgebildeten rhombischen Krystalle zeigen Löslichkeitsverhältnisse, die denen der inaktiven Base gleichen. Die Analysen führen zur Formel $\mathrm{G}_{21} \mathrm{H}_{23} \mathrm{NO}_{4}$, die gleich der der inaktiven Base aufzulösen ist in $\mathrm{C}_{19} \mathrm{H}_{15} \mathrm{O}\left(\mathrm{NCH}_{3}\right)(\mathrm{OH})_{2}\left(\mathrm{OCH}_{3}\right)$. Das Alkaloid scheint also isomer mit Bebirin zu sein und ich bezeichne es daher einstweilen als Isobebirin.

Der Rest des Basengemenges, wie er aus dem amorphen Bebirinum sulfuricum zurückbleibt, zeigt die bereits von Scholtz angeführten unangenehmen Eigenschaften. Ich habe ihn bis jetzt noch nicht näher untersucht.

\section{Untersuchungen über das $\beta$-Bebirin.}

Die Untersuchungen, die über die Konstitution des Hauptalkaloids, des $\beta$-Bebirins, angestellt wurden, klärten die Funktion der vier Sauerstoffe im Molekül desselben auf. Das Bebirin enthält, wie schon gesagt, e in phenolisches Hydroxyl, wie die Löslichkeit in Alkali und die Bestimmung nach Zerewit in off zeigt. Die Acetylierung, die nach Scholtz durch kurzes Erhitzen mit Essigsäureanhydrid auf $60^{\circ}$ vorgenommen wurde, führte zu einem Monoacetylderivat. Die Benzoylierung nach Scholtz durch Schmelzen mit Benzoësäureanhydrid greift aber tiefer in den Bau des Alkaloids ein, indem das Verhalten gegen Alkali und verdünnte Säure, in welcher das Reaktionsprodukt unlöslich 
ist, sowie die Analysen ergaben, daß nicht nur das phenolische Hydroxyl, sondern auch unter Aufspaltung des $\mathrm{N}$-haltigen Ringes der Stickstoff benzoyliert wurde. ${ }^{1}$ Außerdem tritt noch eine dritte verseifbare Benzoylgruppe ein, jedenfalls unter Öffnung einer der oxydartigen Sauerstoffbindungen, deren Vorhandensein später besprochen wird.

Eine Carbonylgruppe konnte durch Einwirkung von Phenylhydrazin auf das Bebirin in essigsaurer Lösung nicht nachgewiesen werden, da das bei längerer Einwirkung entstehende Reaktionsprodukt sich aus 1 Molekül Phenylhydrazin und 2 Molen Bebirin bildete, wie die Stickstoffbestimmung zeigte. ${ }^{2}$ Es ist also ein komplizierter Reaktionsverlauf anzunehmen.

Die Versuche, das Bebirin zu methylieren, brachte die Aufklärung über die Natur der beiden restlichen O-Atome. Mit Nitrosomethylharnstoff ${ }^{3}$ konnte das Methylbebirin erhalten werden. Allerdings traten auch hier Nebenreaktionen auf, die die Ausbeute auf $60 \%$ herabsetzten.

Ganz anders ist der Reaktionsverlauf bei der Behandlung des Bebirins mit Dimethylsulfat. Wenn man die Base mit 3 Molen Dimethylsulfat und 6 Molen wässeriger $\mathrm{KOH}$ am Wasserbad erwärmt bis zum Verschwinden des Öls, so enthält die Lösung ein quartäres Sulfalt und es konnte nach dem Ansäuern die Base mit $\mathrm{Na}_{2} \mathrm{CO}_{3}$ nicht gefällt werden. Durch konzentrierte JK-Lösung wurde daher das Jodid gefällt, welches in $\mathrm{KOH}$ leicht löslich ist. Wie auch die kombinierte Methoxyl- und Methylimidbestimmung zeigte, ist also bloß Methylierung des Stickstoffs, nicht aber des Hydroxyls eingetreten. Ähnliche Beobachtungen

1 Vgl. Pschorr, Ber. Deutsch. chem. G., 35, 4384 (1902), bezüglich des Amorphins, und Gadamer, Arch. f. Pharm., 249, 602 (1911), bezüglich des Bulbocapnins.

$20 \cdot 2400 \mathrm{~g}$ Substanz gaben bei $b=737 \cdot 5 \mathrm{~mm}$ und $t=20^{\circ} 14 \cdot 40 \mathrm{~cm}^{3} \mathrm{~N}$, entsprechend $6 \cdot 69 \% \mathrm{~N}$.

$0.2642 \mathrm{~g}$ Substanz gaben bei $b=756.0^{\prime \prime} \mathrm{mm}$ und $t=19^{\circ} 14.50 \mathrm{~cm}^{3} \mathrm{~N}$, entsprechend $6 \cdot 30 \% \mathrm{~N}$.

Ber. für $\mathrm{C}_{21} \mathrm{H}_{23} \mathrm{NO}_{4} 3 \cdot 970 /_{0} \mathrm{~N}$, fül: $\mathrm{C}_{21} \mathrm{H}_{23} \mathrm{NO}_{3} \cdot \mathrm{N}_{2} \mathrm{HC}_{6} \mathrm{H}_{5}=\mathrm{C}_{2 i} \mathrm{H}_{29} \mathrm{~N}_{3} \mathrm{O}_{3}$ $9 \cdot 48 \% \%_{0} \mathrm{~N}$.

3 Fr. P. 374378; vgl. Hans Meyer, Analyse und Konstitutionsermittlung organischer Verbindungen, 478. 
bezüglich des a-Bebirins und des Chondodrins machte auch Scholtz (1. c.).

Auf andere und sehr interessante Weise verläuft die Einwirkung des Dimethylsulfats, wenn man genau die Arbeitsweise von Pschorr ${ }^{1}$ einhält, also bei langsamer Einwirkung von 5 Molen Dimethylsulfat und 4.3 Molen Alkali in wässeriger Lösung bei $0^{\circ}$. Das Reaktionsprodukt, das als Jodid mit konzentrierter JK-Lösung gefällt wurde, zeigte keine Löslichkeit in Alkali mehr, so daß also Methylierung des phenolischen Hydroxyls eingetreten war. Das Salz ist quartärer Natur, denn aus der wässerigen Lösung wird die Base mit $\mathrm{Na}_{2} \mathrm{CO}_{3}$ oder $\mathrm{NaOH}$ nicht gefällt und läßt sich auch nicht durch $\mathrm{CHCl}_{3}$ aus der alkalischen Lösung ausschütteln. Trotzdem ist keine Methylierung am Stickstoff eingetreten, wie die Methylimidbestimmung zeigte, so daß die quartäre Bindung des Stickstoffs nur durch Umlagerung im Molekül entstanden sein kann.

Die Reaktion verläuft also beim Arbeiten in der Hitze unter Methylierung des Stickstoffs allein, bei der langsamen Einwirkung bei $0^{\circ}$ unter Methylierung des Sauerstoffs allein, wobei trotzdem der Stickstoff quartäre Funktion annimmt.

Die Verbrennung dieses Jodids nun, das bei $130^{\circ}$ getrocknet wurde, um Krystallwasser auszuschließen, ergab das Eintreten von $2 \mathrm{H}_{2} \mathrm{O}$ in das Molekül, was nur so zu erklären ist, daß die beiden restlichen $\mathrm{O}$-Atome oxydartig gebunden waren, welche Bindung unter diesen Versuchsbedingungen unter Aufnahme von $2 \mathrm{H}_{2} \mathrm{O}$ gespalten wurde. Die Bestimmung nach Zerewitinoff ergab als Bestätigung die Anwesenheit von vier Hydroxylen im Molekül.

Ein ähnlicher Übergang vom tertiären Bebirin in eine quartäre Verbindung wird im experimentellen Teil beim Verhalten des inaktiven Umwandlungsproduktes des Bebirins beschrieben werden.

Da diese Arbeit von der Voraussetzung der genetischen Zusammengehörigkeit auch dieser Alkaloide mit der hypothetischen Stammsubstanz von der Konstitution

1 Ber. Deutsch. chem. G., 44, 2633 (1911) 


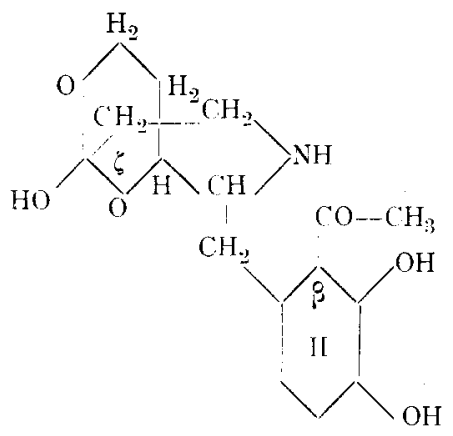

ausgehend in Angriff genommen wurde, ist es vielleicht gestattet, auch auf Grund dieser noch dürftigen Resultate ein Bild von der Konstitution des Bebirins $z u$ geben, das natürlich nur ein annäherndes sein kann und mit allem Vorbehalt gegeben wird.

Da dem Gerüst des Bebirinmoleküls die C-Anzahl $19 \mathrm{zu}$ kommt, ist die Seitenkette der Stammsubstanz in $\beta$, die in den verschiedensten Stadien des Abbaus beobachtet wird, noch um eine $\mathrm{CH}_{2}$-Gruppe reicher anzunehmen. ${ }^{1}$ Die beiden Hydroxyle des Kernes II, die bis jetzt in allen Alkaloiden dieser Gruppe beobachtet wurden, sind jedenfalls auch im Bebirin vorhanden. Eine davon ist in diesen methyliert. Im Isobebirin, das die grüne Farbenreaktion mit $\mathrm{FeCl}_{3}$ zeigt und das zwei Hydroxylgruppen aufweist, sind vielleicht beide frei. Ein Beweis des Vorhandenseins dieser beiden OH-Gruppen ist auch die Bildung von Protocatechusäure bei der Kalischmelze, die allerdings am Alkaloid $B$ beobachtet wurde, aber bei der Ähnlichkeit im Bau der Basen, die sich in derselben Pflanze bilden, wohl ohne weiteres auch auf das Bebirin zu übertragen ist. Von den drei Sauerstoffen des hydrierten Kernes I der Stammsubstanz ist im Bebirin wahrscheinlich nur einer vorhanden, da $\alpha$-Bebirin bei der Zinkstaubdestillation $o$-Kresol liefert, wie Scholtz nachgewiesen hat; so ist also auch der zweite der beiden dieser Gruppe von Alkaloiden charakteristischen Benzolkerne nachgewiesen, und zwar mit dem Sauerstoff in der Stellung $\zeta$, wie er sich auch im

1 Auch im Molekül des Corycavins $\mathrm{C}_{23} \mathrm{H}_{21} \mathrm{NO}_{6}$ (zwei Oxymethylen-, einc Methylimidgruppe) dürtte in ähnlicher Weise eine längere Seitenkette anzunehmen sein. Gäbe1, Arch. für Pharm., 248, 214 (1910). 
Narkotin findet. Dieser Sauerstoff ist oxydartig gebunden und $z$ war, wie aus der leichten Aufspaltbarkeit des Sauerstoffringes unter $\mathrm{H}_{2} \mathrm{O}$-Aufnahme wohl folgt, nicht in einem fünf-oder sechsgliedrigen Ring. Der vierte ebenfalls oxydartig gebundene Sauerstoff wird wohl von der Seitenkette in $\beta$ geliefert, so dab wir uns folgende Vorstellung von der Bildung des Bebirins aus der Stammsubstanz machen können:<smiles>CCCCCNC(CC(O)C(O)C(O)CC)CC1CCC(O)C(O)C1OCC</smiles><smiles>CCOC(=O)C1C(CNCC2C(O)C3[IH]CCC23)CCC(O)C1O</smiles>

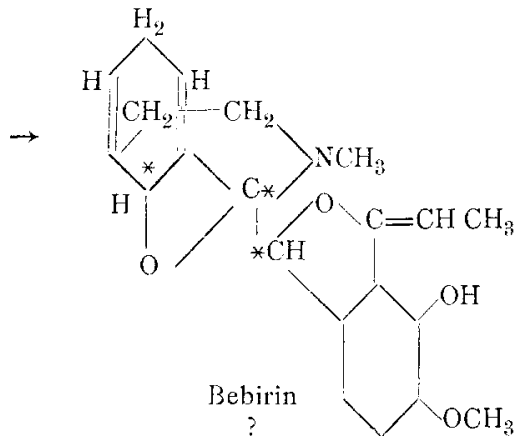

$\mathrm{Ob}$ die labile Gruppierung des $-\mathrm{CH}_{2}-\mathrm{CH}_{2}-\mathrm{N}$-homplexes wie im Morphin auch hier erhalten ist oder ob der Ubergang zum Isochinolinring wie in den übrigen Alkaloiden dieser Gruppe bereits eingetreten ist, ist natürlich unentschieden. Die leichte Abspaltbarkeit des ganzen Komplexes bei der Zinkstaubdestillation spricht vielleicht für erstere Annahme. Ebenso ist die Lage der Methoxylgruppe sowie die zweite Haftstelle der beiden ätherartig gebundenen Sauerstoffe willkürlich angenommen.

Für diese Formulierung sprechen auch die Eigenschaften des Produktes, das bei der Methylierung nach Pschorr entsteht. 
Es muß ihm bei Zugrundelegung der oben entwickelten Formel des Bebirins folgende Struktur zukommen:

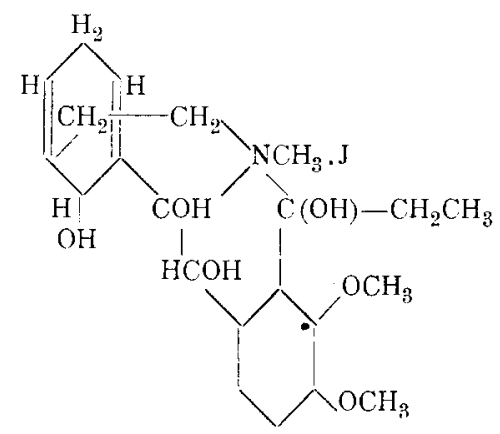

Daß der Kern I hydroaromatisch ist, folgt daraus, daß der Körper in $\mathrm{KOH}$ unlöslich ist, also keine phenolischen Eigenschaften mehr besitzt, nachdem die eine in Bebirin ursprünglich vorhandene Phenolgruppe methyliert wurde. Durch die hydrolytische Aufspaltung bildeten sich also nur alkoholische Hydroxyle, die der Methylierung mit Dimethylsulfat nicht zugänglich sind. Die Entstehung der quartären Bindungsweise des Stickstoffs unter Erhaltung aller Hydroxyle, also ohne $\mathrm{H}_{2} \mathrm{O}-\mathrm{Ab}$ spaltung, kann auch nur auf diese Weise erklärt werden: Es tritt zuerst Ringschließung unter Anlagerung des Stickstoffs an die doppelte Bindung der Seitenkette (unter dem Einfluß der Methylschwefelsäure?) ein, worauf dann die Sauerstoffbrücke unter Aufnahme von $\mathrm{H}_{2} \mathrm{O}$ sich löst. Diese Beteiligung des Stickstoffs an der Bildung zweier Ringsysteme findet ihre Analogie im Molekül des Berberins und des Corydalins.

In der aufgestellten Formel sind drei asymmetrische C-Atome vorhanden, wodurch das Auftreten mehrerer Stereoisomeren des Bebirins erklärt ist.

\section{Experimenteller Teil.}

\section{Darstellung der Alkaloide.}

$200 \mathrm{~g}$ Bebirinum sulfuricum amorphum von Merck wurden in $\mathrm{H}_{2} \mathrm{O}$ gelöst, in welchem sie leicht unter Bildung einer dunkelbraun gefärbten Flüssigkeit in Lösung gehen. Auf Zusatz von 
etwas mehr als der berechneten Menge $\mathrm{Na}_{2} \mathrm{CO}_{3}$-Lösung fiel ein voluminöser, graubraun gefärbter Niederschlag sofort aus, der abgesaugt und durch Aufstreichen auf Tonteller bei Zimmertemperatur getrocknet $(A)$ wurde. Ausbeute zirka $90 \%$.

$4 \cdot 3 g$ fielen als gelbe Flocken erst bei längerem Stehen der alkalischen Mutterlage aus. Daraus wurde mit Benzol $1 g$ ¡-Bebirin extrahiert; nach dem Ausziehen mit Alkohol blieben $1 \cdot 1 \mathrm{~g}$ Isobebirin als weißes, fast reines Pulver zurück. Der Alkoholauszug enthielt ein Gemenge von Basen, als Hauptbestandteil wahrscheinlich das Alkaloid $B$.

Die noch dunkel gefärbte Mutterlauge enthielt noch reichlich Alkaloide, da mit $\mathrm{J}_{2} \mathrm{JK}$-Lösung ein beträchtlicher Niederschlag ausfiel. Doch konnte daraus keine einheitliche Base gewonnen werden.

Der Niederschlag $A$ wurde 7 mal mit zirka $750 \mathrm{~cm}^{3}$ Benzol ausgekocht bis zur Erschöpfung. So wurden $40 \mathrm{~g} \beta$-Bebirin fast rein gewonnen. Der Rückstand wurde 4 mal mit $1 \frac{1}{2} l$ Alkohol in der Siedehitze ausgezogen. Der erste Auszug ließ beim Erkalten keinen Niederschlag fallen; durch Versetzen mit $\mathrm{H}_{2} \mathrm{O}$ im Überschuß nach stärkerem Einengen wurden $20 \mathrm{~g}$ gefällt, die nochmals in Alkohol gelöst und mit $\mathrm{H}_{2} \mathrm{O}$ zur Ausscheidung in Form eines fein verteilten gelben Niederschlages gebracht wurden. Auf diese Weise erhielt ich $9.5 \mathrm{~g}$ vom Alkaloid $B$.

Der zweite Auszug gab beim Erkalten einen Niederschlag, der aus 3.9 noch bräunlich gefärbtem Isobebirin bestand. Mit Wasser wurden nach dem Einengen $5.5 \mathrm{~g}$ gefällt, die zur Hauptmenge aus Alkaloid $B$ bestanden.

Der dritte alkoholische Extrakt endlich ergab beim $\mathrm{Ab}$ kühlen $1 \cdot 6 g$ weißes, fast reines Isobebirin, das in reinem Alkohol fast un!öslich war.

Durch Fällen mit Wasser wurde aus diesem und dem vierten Auszug nur mehr eine sehr kleine Fällung erhalten. Durch Eindampfen der verschiedenen wässerig-alkoholischen Mutterlaugen wurden bloß dunkle, stark verschmierte Rückstände gewonnen.

Aus dem nach dem Ausziehen mit Alkohol bleibenden Rückstand konnten keine einheitlichen Fraktionen mehr erzielt werden. Er löst sich beträchtlich in Wasser unter Bildung einer fast schwarz gefärbten Lösung, ist aber in verdünnten Säuren auch 
nur mehr zum Teil löslich, was auf eine während der Trennung der Basen vor sich gehende nachträgliche Veränderung, wahrscheinlich Oxydation durch den Luftsauerstoff, schließen läßt. ${ }^{1}$ Auch in Pyridin, sonst ein ausgezeichnetes Lösungsmittel für alle Alkaloide, bleibt er zum großen Teile ungelöst.

Bei einem früheren Versuche wurde, wie schon erwähnt, das Basengemenge mit Äther extrahiert. Dabei erhält man aber nicht reines $\beta$-Bebirin, sondern es wird auch ein im Benzol unlöslicher Anteil ausgezogen (zirka $20 \%$ ), der sich in Methylalkohol nur teilweise löst.

\section{B-Bebirin.}

Die Reinigung desselben wurde, wie bereits mitgeteilt durch wiederholtes Lösen im Benzol und fraktioniertes Fällen, mit Petroläther erreicht. Die Verbrennungen, an verschiedenen Fraktionen vorgenommen, ergaben stets übereinstimmende Werte. Auch die Salze sind amorph und können nur durch Eindampfen der neutralisierten Lösungen gewonnen werden als glasartiger Rückstand. Das Jodid, das durch Fällen mit konzentrierter JK-Lösung als weißer flockiger Niederschlag erhalten wurde, zeigt einen Zersetzungspunkt von $245^{\circ}$ bei raschem Erhitzen.

Das lufttrockene Bebirin gibt beim Erhitzen auf $100^{\circ}$ eine Gewichtsabnahme, die ungefähr $1 \mathrm{Mol} \mathrm{H} \mathrm{H}_{2} \mathrm{O}$ entspricht, so bei den ersten drei Analysen $7 \cdot 19,6 \cdot 91,5 \cdot 10 \%$, berechnet $5 \cdot 10 \%$.

I. $0.2602 g$ bei $100^{\circ}$ getrocknete Substanz gaben $0.6808 \mathrm{~g} \mathrm{CO}_{2}$ und

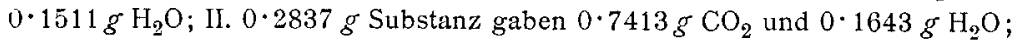
III. $0.2801 \mathrm{~g}$ Substanz gaben $0.7353 \mathrm{~g} \mathrm{CO}_{2}$ und $0 \cdot 1632 \mathrm{~g} \mathrm{H}_{2} \mathrm{O}$; IV. $0.2054 \mathrm{~g}$ Substanz gaben $0.5369 \mathrm{~g} \mathrm{CO}_{2}$ und $0.1196 \mathrm{~g} \mathrm{H} \mathrm{H}_{2} \mathrm{O} ; \mathrm{V} .0 .5690 \mathrm{~g}$ Substanz gaben bei $b=752 \mathrm{~mm}$ und $t=22^{\circ} 22 \cdot 50 \mathrm{~cm}^{3} \mathrm{~N}$; VI. $0 \cdot 3288 \mathrm{~g}$ Substanz gaben $0.2766 \mathrm{~g}$ $\mathrm{JAg}$ bei der Methoxyl- und $0.2006 \mathrm{~g} \mathrm{JAg}$ bei der Methylimidbestimmung; VII. $0.2364 \mathrm{~g}$ Substanz gaben nach Zerewitinoff bei $b=737 \mathrm{~mm}$ und $t=21 \cdot 5^{\circ} 18 \cdot 0 \mathrm{~cm}^{3} \mathrm{CH}_{4}$.

Gef. $\%$ C I. $71 \cdot 36$, II. $71 \cdot 26$, III. $71 \cdot 59$, IV. $71 \cdot 30$. Ber. $71 \cdot 35$.

${ }_{10}^{0}$ H I. $6 \cdot 46$, II. $6 \cdot 44$, IV. $6 \cdot 47$, IV. $6 \cdot 47$. $6 \cdot 56$.

$0_{0}$ N V. $4 \cdot 47$. $3 \cdot 97$.

$0: \mathrm{CH}_{3}$ an $\mathrm{O}$ VI. $5 \cdot 38$
$0: \mathrm{CH}_{3}$ an N VI. $3 \cdot 91,9 \cdot 29$.

$0: 0 H$ VII $5 \cdot 12$. $4 \cdot 82$.

1 Vgl. Scholt $z$, Archiv f. Pharm., 249, 409 (1911). 
Die Analysen ergaben also die Formel

$$
\mathrm{C}_{21} \mathrm{H}_{23} \mathrm{NO}_{4}=\mathrm{C}_{19} \mathrm{H}_{16} \mathrm{O}_{2}\left(\mathrm{NCH}_{3}\right) \cdot(\mathrm{OH}) \cdot\left(\mathrm{OCH}_{3}\right) \text {. }
$$

Das Molekulargewicht berechnet sich zu 353.21, die Bestimmung desselben nach der Siedepunktserhöhungsmethode mit $\mathrm{CHCl}_{3}(k=36 \cdot 6)$ ergab $361 \cdot 3$.

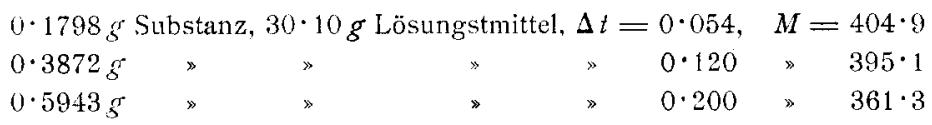

Optisches Verhalten des $\beta$-Bebirins: Die Lösung von $0.7591 \mathrm{~g}$ in Pyridin, deren Gewicht $44.710 \mathrm{~g}$ betrug ( $p=1 \cdot 70$, $d=0.999)$, zeigte bei $21^{\circ}$ im Dezimeterrohre eine Drehung von $-0 \cdot 42^{\circ}$. Hieraus berechnet sich $[a]_{D}^{21}=-24 \cdot 7$ in Pyridin.

$0.6020 \mathrm{~g}$ Bebirin, in absolutem Alkohol gelöst (Gewicht der Lösung $36 \cdot 726 g, p=1 \cdot 64 . d=0 \cdot 810$ ) zeigten eine Drehung von $+0.38^{\circ}$; also $[\alpha]{ }_{D}^{21}=+28.6$ in absolutem Alkohol.

\section{Alkaloid $B$.}

Die Base wurde durch öfteres Lösen in Alkohol und Fällen mit Wasser in reinem Zustand mit konstanten Verbrennungswerten erhalten. Sie bildet dann ein hellgelbes Pulver, das sich unter dem Polarisationsmikroskop als krystallinisches Aggregat von unregelmäßig begrenzten, doppelbrechenden, kugelförmig bis elliptischen Gebilden erweist.

I. $0.2698 \xi$ bei $100^{\circ}$ getrockneter Substanz gaben $0.6849 g \mathrm{CO}_{2}$ und $0.1459 g \mathrm{H}_{2} \mathrm{O}$; II. $0 \cdot 2870 \mathrm{~g}$ Substanz gaben $0.7321 \mathrm{~g} \mathrm{CO}_{2}$ und $0.1608 g \mathrm{H}_{2} \mathrm{O}$; III. $0.2452 \mathrm{~g}$ Substanz gaben $0.6234 \mathrm{~g} \mathrm{CO}_{2}$ und $0.1305 \mathrm{~g} \mathrm{H}_{2} \mathrm{O}$; IV. $0.1836 \mathrm{~g}$ Substanz gaben $0.4667 \mathrm{~g} \mathrm{CO}_{2}$ und $0.0998 \mathrm{~g} \mathrm{H}_{2} \mathrm{O}$; V. $0.1665 g$ Substanz gaben $0.4231 \mathrm{~g} \mathrm{CO}_{2}$ und $0.0899 \mathrm{~g} \mathrm{H}_{2} \mathrm{O}$; VI. $0.4596 \mathrm{~g}$ Substanz gaben bei $b=738 \mathrm{~mm}$ und $t=20^{\circ} 16.50 \mathrm{~cm}^{3} \mathrm{~N}$; VII. $0.3383 \mathrm{~g}$ Substanz gaben $0.2195 \mathrm{~g} \mathrm{JAg}$ bei der Methoxyl- und $0.2070 \mathrm{~g} \mathrm{JAg}$ bei der Methylimidbestimmung; VIII. $0 \cdot 1108 \mathrm{~g}$ Substanz gaben nach Zerewitinoff bei $b=752 \mathrm{~mm}$ und $t=21^{\circ} 12 \cdot 6 \mathrm{~cm}^{3} \mathrm{CH}_{4}$.

Gef, $\%_{0}$ C I. $69 \cdot 24$, II. $69 \cdot 57$, III. $69 \cdot 34$, IV. $69 \cdot 33$, V. $69 \cdot 30$. Ber. $69 \cdot 26$. $0 / 0$ H I. $6 \cdot 01$, II. $6 \cdot 46$, III. $5 \cdot 91$, IV. $6 \cdot 04$, V. $6 \cdot 00$. $6 \cdot 08$.

$\%$ N VI. $4 \cdot 01$.

$3 \cdot 68$.

$\left.\begin{array}{l}0 \mathrm{CH}_{3} \text { an } \mathrm{O} \text { VII. } 4 \cdot 14 \\ 0 \mathrm{CH}_{3} \text { an N VII. } 3 \cdot 91\end{array}\right\} 8 \cdot 05$.

$7 \cdot 87$.

$\%$ OH VIII. $7 \cdot 82$.

$8 \cdot 92$.

Die hieraus abgeleitete Formel ist

$$
\mathrm{C}_{22} \mathrm{H}_{23} \mathrm{NO}_{5}=\mathrm{C}_{20} \mathrm{H}_{15} \mathrm{O}_{2}\left(\mathrm{NCH}_{3}\right)(\mathrm{OH})_{2}\left(\mathrm{OCH}_{3}\right) \text {. }
$$


Optisches Verhalten des Alkaloids $B: 0 \cdot 5263 \mathrm{~g}$, in Pyridin gelöst (Gewicht der dunklen Lösung 49.236 $g, p=1 \cdot 07$, $d=0.997)$, zeigten eine Drehung des polarisierten Lichtstrahls von $+0.605^{\circ}$, wobei Auerlicht als Lichtquelle benützt werden mußte, also $[\alpha]_{j}^{20}=+56 \cdot 7$.

$2 \cdot 5 \mathrm{~g}$ Alkaloid $B$ wurde mit $25 \mathrm{~g}$ befeuchtetem Ätzkali bei zirka $260^{\circ} 20$ Minuten lang im Silbertiegel geschmolzen, wobei starker Methylamingeruch auftrat. Die dunkle Schmelze wurde nach dem Abkühlen in verdünnter $\mathrm{H}_{2} \mathrm{SO}_{4}$ gelöst und ausgeäthert. Nach dem Verjagen des Äthers hinterblieb ein brauner verschmierter Rückstand, intensiv phenolisch riechend (vielleicht $o$-Kresol?). Nach einiger Zeit bildeten sich daneben schöne Krystalle aus. Mit wenig Wasser versetzt, ging alles in Lösung bis auf eine kleine Menge von Krystallen, die bei 196 bis $197^{\circ}$ (unkorr.) unter Zersetzung schmolzen. Sie zeigten die charakteristische Farbenreaktion der Protocatechusäure mit $\mathrm{FeCl}_{3}$ und verdünnter $\mathrm{Na}_{2} \mathrm{CO}_{3}$-Lösung. Der Mischschmelzpunkt mit reiner Protocatechusäure gab keine Depression. Die braune Lösung zeigte auch intensive Trübgrünfärbung $\mathrm{mit}^{\mathrm{FeCl}} \mathrm{F}_{3}$ und Rotbraunfärbung mit $\mathrm{Na}_{2} \mathrm{CO}_{3}$-Lösung und enthielt wahrscheinlich Brenzcatechin, da alkalische Kupferlösung in der Siedehitze stark reduziert wurde.

\section{Isobebirin.}

Die Base wurde durch Auskochen mit Alkohol von den Beimengungen befreit und durch Eindampfen der mit Methylalkohol versetzten Pyridinlösung in dichten Krystalldrusen gewonnen. Herr Dozent Dr. Emil Dittler am I. mineralogischen Institut der Universität Wien hatte die Güte, ihr Verhalten unter dem Polarisationsmikroskop zu untersuchen, wofür ich ihm auch an dieser Stelle bestens danke. Rhombische Krystalle, gewöhnlich nur von den drei Pinakoiden begrenzt. Gerade Auslöschung; optischer Charakter positiv; starke Dispersion $\rho<v$. Achsenwinkel scheint kleiner zu sein als beim aktiven Isobebirin; Zwillinge; positive Spärolithe bei raschem Krystallisieren. Farbe hellgelb.

I. $0.2328 \mathrm{~g}$ bei $100^{\circ}$ getrockneter Substanz gaben $0.6035 \mathrm{~g} \mathrm{CO}_{2}$ und $0.1325 \mathrm{~g} \mathrm{H}_{2} \mathrm{O}$; II. $3.1988 \mathrm{~g}$ Substanz gaben $0.5146 \mathrm{~g} \mathrm{CO}_{2}$ und $0.1158 \mathrm{~g}$ $\mathrm{H}_{2} \mathrm{O}$; III. $0 \cdot 2870 \mathrm{~g}$ Substanz gaben $0.2356 \mathrm{~g} \mathrm{JAg}$ bei der Methoxyl- und 
$0.1880 g$ bei der Methylimidbestimmung; IN. $0.1512 g$ Substanz gaben hei $l^{\prime}=752 \mathrm{~mm}$ und $t=21^{\circ} 17 \cdot 20 \mathrm{~cm}^{3} \mathrm{CH}_{4}$.

Gef.: I. $70 \cdot 70 \%$ C, $6 \cdot 32 \%$ H, II. $70 \cdot 60 \%$ C, $6 \cdot 470 \%$ H, III. $9 \cdot 43 \%$ $\mathrm{CH}_{3}(5 \cdot 25 \%$ an $\mathrm{O}$ und $4.18 \%$ an $\mathrm{N})$, IV. $7 \cdot 82 \% \mathrm{OH}$.

Bezuiglich der Verbrennungen sei auf die Anmerkung p. 877 verwiesen. Die Wägung des Rückstandes $(0.0020 \mathrm{~g})$ wurde bei Verbrennung II vorgenommen, wodurch sich $71 \cdot 32$, beziehungsweise $71 \cdot 16 \% \mathrm{C}$ berechnen, je nachdem man annimmt, daß $\mathrm{Na}_{2} \mathrm{CO}_{3}$ oder $\mathrm{Na}-\mathrm{Sal} z$ des Alkaloids die Verunreinigung war.

Über das optische Verhalten der Base und ihr Verhältnis zum Alkaloid, das ich aus Bebirinum sulfuricum crystallisatum von Merck dargestellt habe, wurde bereits gesprochen.

Dieses Alkoloid wurde daraus dadurch rein gewonnen, daß die beim Versetzen der wässerigen Sulfatlösung mit $\mathrm{NH}_{3}$ ausfallende Base mit $\mathrm{CHCl}_{3}$ ausgeschüttelt wurde und der nach dem Vertreiben des $\mathrm{CHCl}_{3}$ bleibende Rückstand nochmals mit $\mathrm{CHCl}_{3}$ in der Hitze gelöst wurde, wobei ein Teil sich als schwerer löslich erwies. Die Lösung wurde mit der dreibis vierfachen Menge Petroläther versetzt, wodurch eine kleine Menge von gefärbter Substanz gefällt wird, und nach dem Filtrieren der langsamen Verdunstung überlassen. Dabei scheiden sich sehr schön ausgebildete, vollständig einheitliche Krystalle, die oft die Länge von $5 \mathrm{~mm}$ erreichen, ab, deren krystallographische Untersuchung ich der Liebenswürdigkeit des Herrn Dozenten Dr. Himmelbauer am II. mineralogischen Institut der Universität verdanke:

Rhombisch, Begrenzung gewöhnlich nur (100) (010) (001), Spaltbarkeit nach zwei Pinakoiden sicher (eine darunter sehr gut), gerade Auslöschung, parallel der besseren Spaltbarkeit $i$ Optischer Charakter positiv, mit einem scheinbaren Achsenwinkel von etwa $60^{\circ}$. Deutliche Dispersion, $p>v$. Häufig Zwillinge. Farbe hellgelb. Bei raschem Krystallisieren positive Sphärolithe.

Diese Krystalle dürften Krystallchloroform enthalten; wenigstens ergab die Bestimmung der Gewichtsabnahme bei $100^{\circ}$ an lufttrockenen Krystallen bei Analyse IV, die einen Tag lang aus der Lösung herausgenommen waren, eine Größe, die zirka dem Gehalt an $1 \mathrm{CHCl}_{3}$ entspricht, nämlich $29 \cdot 70 \%$ gegen $33.79 \%$ berechnet. Der Gehalt nimmt rasch ab. Beim vollständigen Eindunsten der abgegossenen Lösung bilden sich 
isotrope Krusten von Oktaedern, die jedenfalls der krystallchloroformfreien Substanz zugehören. Denn sie bilden sich auch aus den einheitlichen rhombischen Krystallen nach dem neuerlichen Auflösen derselben unter dieser Bedingung. Die rhombischen Krystalle lösen sich leicht in $\mathrm{CHCl}_{3}$, beim Trocknen bei $100^{\circ}$ zerfallen sie und sind in $\mathrm{CHCl}_{3}$ bedeutend schwerer löslich. Beim Erwärmen mit Alkohol oder Essigäther gehen sie anscheinend in eine amorphe Modifikation über, nämlich in weiße Flocken.

I. $0.1538 \mathrm{~g}$ Substanz, bei $100^{\circ}$ getrocknet (Gewichtsabnahme $0.0206 \mathrm{~g}$ ), gaben $0.4016 g \mathrm{CO}_{2}$ und $0.0886 g \mathrm{H}_{2} \mathrm{O}$; II. $0.1574 g$ Substanz $(-0.0222 g)$

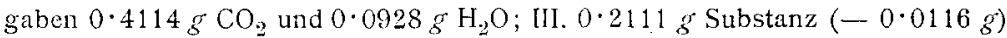
gaben $0.5506 \mathrm{~g} \mathrm{CO}_{2}$ und $0.1197 \mathrm{~g} \mathrm{H}_{2} \mathrm{O}$; IV. $0.1633 \mathrm{~g}$ Substanz $(-0.0485 \mathrm{~g}$ ) gaben $0.4293 \mathrm{CO}_{2}$ und $0.0939 \mathrm{~g} \mathrm{H}_{2} \mathrm{O} ; \mathrm{V} .0 .2139 \mathrm{~g}$ Substanz $(-0.0310 \mathrm{~g}$ ) gaben $0.5599 \mathrm{~g} \mathrm{CO}_{2}$ und $0.1257 g^{\circ} \mathrm{H}_{2} \mathrm{O}$; VI. $0 \cdot 3792 g$ Substanz $(-0.0486 g)$ gahen bei $b=751 \mathrm{~mm}$ und $t=21^{\circ} 15 \cdot 6 \mathrm{~cm}^{3} \mathrm{~N}$; VII. $0.2432 g$ Substanz gaben $0 \cdot 1671 \mathrm{~g}$ JAg bei der Methoxylbestimmung; VIII. $0 \cdot 1710 \mathrm{~g}$ Substanz gaben 0.2141 bei der Methoxyl- und Methylimidbestimmung; IX. $0 \cdot 1278$ is Substanz gaben nach Zerewitinoff bei $b=746 \mathrm{~mm}$ und $t=17 \cdot 5^{\circ}$ $13 \cdot 30 \mathrm{~cm}^{3} \mathrm{CH}_{4}$.

Gef, $0 \%$ C I. $71 \cdot 21$, II. $71 \cdot 29$, III, $71 \cdot 13$, IV. $71 \cdot 70$, V. $71 \cdot 46$. Ber. $71 \cdot 35$. $\%$ H I. $6 \cdot 40$, II. $6 \cdot 55$, III. $6 \cdot 30$, IV. $6 \cdot 35$, V. $6 \cdot 54$. $0 / 0$ N VI. $4 \cdot 66$ $3 \cdot 97$. $0 / 0 \mathrm{CH}_{3}$ an $\mathrm{O}$ VII. $4 \cdot 39$. $4 \cdot 26$. $0 \mathrm{C}_{0} \mathrm{CH}_{3}$ an $\mathrm{O}$ und $\mathrm{N}$, VIII. $8 \cdot 01$. $8 \cdot 51$. $0 \%$ OH IX. $7 \cdot 20$. $9 \cdot 63$.

Die Formel des Isobebirins ist also

$$
\mathrm{C}_{21} \mathrm{H}_{23} \mathrm{NO}_{4}=\mathrm{C}_{19} \mathrm{H}_{15} \mathrm{O}\left(\mathrm{NCH}_{3}\right)(\mathrm{OH})_{2}\left(\mathrm{OCH}_{3}\right) \text {. }
$$

Optisches Verhalten: 0.7330 g Substanz, in Pyridin gelöst (Gewicht der Lösung $42 \cdot 373 g, p=1 \cdot 73, d=0 \cdot 980$ ), drehten im Dezimeterrohr den polarisierten Lichtstrahl um $-0.81^{\circ}$. Daraus berechnet $\operatorname{sich}[\alpha]_{D}=-47 \cdot 7$ bei $22^{\circ}$.

Um die Anwesenheit von zwei phenolischen $\mathrm{OH}$-Gruppen im Isobebirin $z u$ beweisen, wurde versucht, mit Nitrosomethylharnstoff ${ }^{1}$ ( 6 Mole) zu einem Dimethylisobebirin zu kommen. Tatsächlich gelang es, aus $1 g$ der Base nach dem Methylieren durch Extrahieren mit Benzol einen krystallisierten Körper vom

1 Fr. P. $3743 r 8$; vgl. Hans Mejer, Analyse und Konstitutionsvermittlung organischer Verbindungen, tis. 
Schmelzpunkte 120 bis $122^{\circ}$ in einer Ausbeute von $0.4 \mathrm{~g} \mathrm{zu}$ gewinnen, der in $\mathrm{KOH}$ unlöslich ist und dessen Methylzahl annähernd auf ein Dimethylisobebirin stimmt.

$0.2296 \mathrm{~g}$ Substanz gaben $0.2472 \mathrm{~g} J \mathrm{Ag}$ bei der Methoxyl- und $0.2605 \mathrm{~g}$ JAg bei der Methylimidbestimmung; gef. $6 \cdot 87+7 \cdot 25 \%=14 \cdot 12 \% \mathrm{CH}_{3}$, ber. fuir $\mathrm{C}_{19} \mathrm{H}_{15} \mathrm{O}\left(\mathrm{NCH}_{3}\right)\left(\mathrm{OCH}_{3}\right)_{3} 11 \cdot 75+3 \cdot 92 \%=15 \cdot 670_{0} \mathrm{CH}_{3}$. Die Methylimidzahl ist viel zu groß; es liegt also wahrscheinlich der Fall vor, daß Methyl vom $\mathrm{O}$ zum $\mathrm{N}$ wandert, beziehungsweise Betainbildung eintritt (vielleicht ist auch zum Teil Verharzung eingetreten, so daß der Rest des Methoxyls erst bei der Methylimidbestimmung angegriffen wurde). Die in diesem Fall nötige Wiederholung der trockenen Destillation konnte infolge Springens des Kölbchens leider nicht gemacht "werden, um das Methyl vollständig zu erhalten.

\section{$\alpha$-Bebirin von Scholtz.}

I. $0 \cdot 1909 g$ Substanz gaben $0.4987 g \mathrm{CO}_{2}$ und $0 \cdot 1084 g \mathrm{H}_{2} \mathrm{O}$; II. $0 \cdot 1175 \mathrm{~g}$ Substanz gaben $0.3072 \mathrm{~g} \mathrm{CO}_{2}$ und $0.0683 \mathrm{~g} \mathrm{H} \mathrm{H}_{2} \mathrm{O}$; III. $11.26 \mathrm{mg}$ Substanz gaben $29.58 \mathrm{mg} \mathrm{CO}_{2}$ und $6.40 \mathrm{mg} \mathrm{H}_{2} \mathrm{O}$; IV. $10.87 \mathrm{mg}$ Substanz gaben $28 \cdot 54 \mathrm{mg} \mathrm{CO}_{2}$ und $6 \cdot 14 \mathrm{mg} \mathrm{H}_{2} \mathrm{O} .1$

Das krystallisierte $\boldsymbol{\alpha}$-Bebirin liefert also dieselben Verbrennungswerte wie das $\beta$-Bebirin.

Gef.: I. $71 \cdot 250 \%$ C, $6 \cdot 31 \%$ H; II. $71 \cdot 30 \%$ C, $6 \cdot 46 \%$ H; III. $71 \cdot 64 \%$ C, $6 \cdot 36 \% \mathrm{H}$; IV. $71 \cdot 61 \% \mathrm{C}, 6 \cdot 32 \% \mathrm{H}$. Ber. für $\mathrm{C}_{21} \mathrm{H}_{23} \mathrm{NO}_{4} 71 \cdot 35 \%$ C, $6 \cdot 56 \% \mathrm{H}$; ber, für $\mathrm{C}_{18} \mathrm{H}_{21} \mathrm{NO}_{3}(\mathrm{Scholtz}) 72 \cdot 24 \% \mathrm{C}, 7 \cdot 02 \%$ H.

Das amorphe Präparat von Scholtz hatte sich, wie bereits gesagt, vollständig umgewandelt. Die Bestimmung der $\mathrm{OH}$-Gruppe nach $\mathrm{Z}$ erewitin off an dem in $\mathrm{CHCl}_{3}$ unlöslichen Anteil zeigte, daß auch hier ähnlich wie bei der analogen Umwandlung des $\beta$-Bebirins Bildung von neuen Hydroxylgruppen eingetreten ist unter Auflösung der oxydartigen Bindung. Der Schmelzpunkt dieser Fraktion lag bei 285 bis $290^{\circ}$ unter Zersetzung bei raschem Erhitzen.

$0.1207 \mathrm{~g}$ Substanz gaben bei $b=747 \mathrm{~mm}$ und $t=23^{\circ} 11.80 \mathrm{~cm}^{3} \mathrm{CH}_{4}$.

Gef. $6 \cdot 63 \%$ OH. Ber. für $\mathrm{C}_{21} \mathrm{H}_{23} \mathrm{NO}_{4} .1 \mathrm{OH} 4 \cdot 82 \%, 2 \mathrm{OH} 9 \cdot 17 \%$.

1 Die dritte und vierte Verbrennung, die mikroanalytisch ausgeführt wurden, verdanke ich der Güte des Herrn Dr. Edelbacher am medizinischchemischen Institut der Universität Innsbruck, dem ich auch an dieser Stelle meinen besten Dank ausspreche. 


\section{Acetylbebirin.}

$1 \mathrm{~g} \beta$-Bebirin wurde in zirka $3 \mathrm{~cm}^{3}$ Essigsäureanhydrid 20 Minuten auf $60^{\circ}$ erwärmt, die dunkelrote Lösung in $\mathrm{H}_{2} \mathrm{O}$ gegossen, in welchem allmählich alles in Lösung ging. Mit Sodalösung wurden dann $0.9 \mathrm{~g}$ flockig gefällt. Das Acetylprodukt ist in $\mathrm{C}_{6} \mathrm{H}_{6}$ noch leichter löslich als $\beta$-Bebirin selbst, amorph, Schmelzpunkt unscharf, 120 bis $142^{\circ}$. Es löst sich in verdünnten Säuren sehr leicht, in Alkali dagegen nicht. Es wurde durch fraktioniertes Fällen mit Petroläther aus der Benzollösung gereinigt.

I. $0 \cdot 1949 \mathrm{~g}$ bei $100^{\circ}$ getrockneter Substanz gaben $0.4988 \mathrm{~g} \mathrm{CO}_{2}$ und $0.1071 \mathrm{~g} \mathrm{H}_{2} \mathrm{O}$; II. $0.4621 \mathrm{~g}$ Substanz wurden in methylalkoholischer Lösung mit $45 \cdot 5 \eta_{10} \mathrm{KOH}$ durch zweistündiges Kochen verseift. Die Lösung wurde mit $\mathrm{H}_{2} \mathrm{O}$ stark verdünnt und mit $31.6 \mathrm{~cm}^{3} \mathrm{n} / \mathrm{H}_{10} \mathrm{H}_{2} \mathrm{SO}_{4}$ zurücktitriert, wobei sich die verseifte Base flockig ausschied. Verbrauch an $\mathrm{KOH} 13.60 \mathrm{~cm}^{3}=0.0759 \mathrm{~g}$ $\mathrm{KOH}\left(1 \mathrm{~cm}^{3} \mathrm{KOH}=0.991 \mathrm{~cm}^{3} \mathrm{H}_{2} \mathrm{SO}_{4}=0.00558 \mathrm{~g} \mathrm{KOH}\right) .1$

Gef.: I. $69 \cdot 80 \% \mathrm{C}, 6 \cdot 11 \% \mathrm{H}$; II. $12 \cdot 59 \% \mathrm{COCH}_{\mathrm{g}}$. Ber. für $\mathrm{C}_{19} \mathrm{H}_{16} \mathrm{O}_{2}$. . $\left(\mathrm{NCH}_{3}\right)\left(\mathrm{OCH}_{3}\right)\left(\mathrm{OCOCH}_{3}\right)=\mathrm{C}_{23} \mathrm{H}_{25} \mathrm{NO}_{5} 69 \cdot 83 \% \mathrm{C}, 6.38 \% \mathrm{H}, 10 \cdot 88 \%$ $\mathrm{COCH}_{3}$.

Aber auch dann, wenn man dieselben milden Versuchsbedingungen einhält, tritt oft weiter gehende Acetylierung ein, jedenfalls unter Öffnung der oxydartigen O-Bindung. So erhielt ich einmal einen amorphen Körper vom Schmelzpunkte 140 bis $165^{\circ}$, da zwei verseifbare und, wie die Verbrennungsdaten ergeben, eine unverseifbare Acetylgruppe, die wahrscheinlich in den Kern getreten war, enthielt. In verdünnter $\mathrm{HCl}$ war er leicht löslich.

I.. $0.1662 g$ bei $100^{\circ}$ getrockneter Substanz gaben $0.4103 g \mathrm{CO}_{2}$ und $0.0868 \mathrm{~g} \mathrm{H} \mathrm{H}_{2} \mathrm{O}$; II. $0.4791 \mathrm{~g}$ Substanz wurden mit $15 \mathrm{~cm}^{3}$ Methylalkohol und $10 \cdot 84 \mathrm{~cm}^{3} \mathrm{n} . \mathrm{NaOH}$ durch dreistündiges Kochen verseift. Die mit $\mathrm{H}_{2} \mathrm{O}$ verdünnte Lösung wurde mit $88.66 \mathrm{~cm}^{3} n / 10 \mathrm{H}_{2} \mathrm{SO}_{4}$ zurücktitriert (Ausfallen der Base). Verbrauch an $\mathrm{NaOH} 1.89 \mathrm{~cm}^{3}=0.07371 \mathrm{~g} \mathrm{NaOH}\left(1 \mathrm{~cm}^{3} \mathrm{NaOH}=9.905 \mathrm{~cm}^{3}\right.$ $\left.\mathrm{H}_{2} \mathrm{SO}_{4}=0 \cdot 0390 \mathrm{~g} \mathrm{NaOH}\right)$.

Gef.: I. $67 \cdot 33 \%$ C, $5 \cdot 80 \% \mathrm{H} ; 16 \cdot 54 \% \mathrm{COCH}_{3}$. Ber. für $\mathrm{C}_{19} \mathrm{H}_{14}$. $. \mathrm{O}\left(\mathrm{NCH}_{3}\right) \mathrm{COCH}_{3}\left(\mathrm{OCOCH}_{3}\right)_{2}\left(\mathrm{OCH}_{3}\right)=\mathrm{C}_{27} \mathrm{H}_{29} \mathrm{NO}_{7} 67.24 \% \mathrm{C}, 6.32 \% \mathrm{H}$, $17 \cdot 96 \% \mathrm{OOCH}_{3}$.

Das verseifte Produkt (Schmelzpunkt 165 bis $195^{\circ}$ ) wies bei der Bestimmung nach Zerewitinoff tatsächlich einen erhöhten Gehalt an $\mathrm{OH}$ auf.

1 Vgl. Vongerichte, A., 294, 215, Best. d. Benzoylmorphins. 
$0.1176 \mathrm{~g}$ Substanz gaben bei $b=752 \mathrm{~mm}$ und $t=21^{\circ} 17 \cdot 2 \mathrm{~cm}^{3} \mathrm{CH}_{4}$, entsprechend $10 \cdot 05 \%$ OH (Bebirin ber. $4.820 \%_{0} \mathrm{OH}$ ).

\section{Benzoylierung des Bebirins.}

$1 g \beta$-Bebirin wurde mit 3 Molen Benzoesäureanhydrid 20 Minuten lang bei $100^{\circ}$ geschmolzen. Die Schmelze wurde mit alkalischem und dann saurem Wasser bei gelinder Temperatur ausgezogen, wobei nur sehr wenig in Lösung ging. Die harzige Masse, die zurückblieb, wurde durch Lösen in Pyridin und Fällen mit Wasser gereinigt. Sie stellte dann ein rötlichweißes Pulver dar, das bei 144 bis $147^{\circ}$ schmolz und weder in $\mathrm{KOH}$ noch verdünnten Säuren, von organischen Lösungsmitteln nur in Pyridin leicht löslich war.

I. $0 \cdot 1815 g$ bei $100^{\circ}$ getrockneter Substanz gaben $0.4877 g \mathrm{CO}_{2}$ und $0.0931 \mathrm{~g} \mathrm{H}_{2} \mathrm{O} ; 0.1874 \mathrm{~g}$ Substanz gaben $0.5042 \mathrm{~g} \mathrm{\textrm {CO } _ { 2 }}$ und $0.0939 \mathrm{~g} \mathrm{H}_{2} \mathrm{O}$; III. $0 \cdot 3059 \mathrm{~g}$ Substanz wurden mit Methylalkohol und $7 \cdot 72 \mathrm{~cm}^{3} \mathrm{n}$. NaOH durch dreistündiges Kochen verseift. Die verdünnte Lösung wurde mit $66 \cdot 35 \mathrm{~cm}^{3}$ $n / 10 \mathrm{H}_{2} \mathrm{SO}_{4}$ zurücktitriert (Ausfallen der Base). Verbrauch an $\mathrm{NaOH} 0.97 \mathrm{~cm}^{3}$ $=0.03783 \mathrm{~g} \mathrm{NaOH}\left(1 \mathrm{~cm}^{3} \mathrm{NaOH}=9.83 \mathrm{~cm}^{3} \mathrm{H}_{2} \mathrm{SO}=0.0390 \mathrm{~g} \mathrm{NaOH}\right)$.

Gef.: I. $73 \cdot 29 \%$ C, $5 \cdot 70 \% \mathrm{H}$; II. $73 \cdot 37 \% \%_{0} \mathrm{C}, 5 \cdot 57 \% \%_{0} \mathrm{H}$; III. $32 \cdot 46 \%$ $\mathrm{COC}_{6} \mathrm{H}_{5}$. Ber. für $\mathrm{C}_{19} \mathrm{H}_{16} \mathrm{O}_{2}\left(\mathrm{NCH}_{3} . \mathrm{COC}_{6} \mathrm{H}_{5}\right)\left(\mathrm{OCOC}_{6} \mathrm{H}_{5}\right)_{2}\left(\mathrm{OCH}_{3}\right)=\mathrm{C}_{42} \mathrm{H}_{37} \mathrm{NO}_{8}$ $73 \cdot 77 \%$ C, $5 \cdot 46 \% \mathrm{H}, 30 \cdot 75 \% \mathrm{COC}_{6} \mathrm{H}_{5}$.

Die Einwirkung von Benzoesäureanhydrid auf das $\beta$-Bebirin ist also nach der Gleichung vor sich gegangen:

$$
\begin{aligned}
& \mathrm{C}_{19} \mathrm{H}_{16} \mathrm{O}_{2}\left(\mathrm{NCH}_{3}\right)(\mathrm{OH})\left(\mathrm{OCH}_{3}\right)+2\left(\mathrm{C}_{6} \mathrm{H}_{5} \mathrm{CO}\right)_{2} \mathrm{O}= \\
& \mathrm{C}_{19} \mathrm{H}_{16} \mathrm{O}_{2}\left(\mathrm{NCH}_{3} \cdot \mathrm{COC}_{6} \mathrm{H}_{5}\right)\left(\mathrm{OCOC}_{6} \mathrm{H}_{5}\right)_{2}(\mathrm{OCH})_{3}+\mathrm{C}_{6} \mathrm{H}_{5} \mathrm{COOH} .
\end{aligned}
$$

Das verseifte Produkt schmolz bei 165 bis $172^{\circ}$ und enthielt nach Zerewitin off zwei Hydroxylgruppen:

$0.0919 \mathrm{~g}$ bei $100^{\circ}$ getrockneter Substanz gaben bei $b=744 \mathrm{~mm}$ und $t=22^{\circ} 9 \cdot 50 \mathrm{~cm}^{3}\left[\mathrm{CH}_{4}\right.$.

Gef. $7 \cdot 03 \%$ OH; ber. für $\mathrm{C}_{19} \mathrm{H}_{16} \mathrm{O}_{2}\left(\mathrm{NCH}_{3} \cdot \mathrm{COC}_{6} \mathrm{H}_{5}\right)(\mathrm{OH})_{2}\left(\mathrm{OCH}_{3}\right)=$ $\mathrm{C}_{28} \mathrm{H}_{29} \mathrm{NO}_{6} 7 \cdot 15 \%$ OH.

\section{$\beta$-Methylbebirin.}

$5 \mathrm{~g}$ Bebirin wurden in $45 \mathrm{~cm}^{3}$ Alkohol gelöst und nach Zusatz von $1.8 g \mathrm{NaOH}$ in wenig Wasser wurden bei $0^{\circ} 5 g$ 
Nitrosomethylharnstoff (zirka 3 Mole) eingetragen. Nach Beendigung der Realstion wurde der Alkohol verjagt und der Rückstand nach Zugabe von etwas $\mathrm{NaOH}$ mit $\mathrm{C}_{6} \mathrm{H}_{6}$ ausgezogen. Auf diese Weise wurden $3.2 \mathrm{~g}$ Methylbebirin erhalten. Der Rest des Reaktionsproduktes war zwar auch methyliert, da er in $\mathrm{KOH}$ unlöslich war, hat aber eine ähnliche Umlagerung in ein schwerer lösliches Derivat erfahren, jedenfalls unter Aufspaltung der oxydartigen Bindungen, wie sie beim Bebirin so häufig beobachtet werden.

Das Methylbebirin ist in Benzol leichter löslich als das $\beta$-Bebirin und krystallisiert beim Verdunsten desselben in schönen Nadeln: "Schwach lichtbrechende Aggregate von Nadeln. Gerade Auslöschung. $\gamma^{\prime}$ in der Längsrichtung; zwillingslamelliert; stark doppelbrechend; optischer Charakter positiv; tetragonal oder hexagonal (Dittler). Schmelzpunkt 81 bis $83^{\circ}$.

$0.1955 \mathrm{~g}$ Substanz gaben $0.2741 \mathrm{~g} \mathrm{~J}$ Ag bei der Methoxyl- und $0 \cdot 1330 \mathrm{~g}$ $\mathrm{JAg}$ bei der Methylimidbestimmung.

Gef. $8 \cdot 95 \% \%_{0} \mathrm{CH}_{3}$ an $\mathrm{O}$ und $4 \cdot 340 \%_{0} \mathrm{CH}_{3}$ an $\mathrm{N}$; ber. für $\mathrm{C}_{19} \mathrm{H}_{16} \mathrm{O}_{2}\left(\mathrm{NCH}_{3}\right)$. $\left(\mathrm{OCH}_{3}\right)_{2}=\mathrm{C}_{22} \mathrm{H}_{25} \mathrm{NO}_{4} 8 \cdot 18 \% \mathrm{CH}_{3}$ an $\mathrm{O}$ und $4 \cdot 090 / 0 \mathrm{CH}_{3}$ an $\mathrm{N}$.

Bei Anwendung von $1 \frac{1}{2}$ Molen Nitrosomethylharnstoff war anscheinend nicht vollständige Methylierung eingetreten, da der in $\mathrm{C}_{6} \mathrm{H}_{6}$ lösliche Anteil $7 \cdot 46 \% \mathrm{CH}_{3}$ an $\mathrm{O}$ und $3 \cdot 88 \%$ $\mathrm{CH}_{3}$ an $\mathrm{N}$ ergab.

\section{Methylierung mit Dimethylsulfat.}

A. Die Analyse des bei der Methylierung in der Hitze erhaltenen Jodids ergab die Anwesenheit eines Methyls an $O$ und zweier Methyle an $\mathrm{N}$ :

$0.2312 g$ bei $100^{\circ}$ getrockneter Substanz gaben $0.0976 \mathrm{~g} \mathrm{JAg}$ bei der Methoxyl- und $0 \cdot 1840+0 \cdot 0385=0.2225 \mathrm{~g} \mathrm{~J} \mathrm{Ag}$ bei der Methylimidbestimmung.

Gef. $2 \cdot 70 \%{ }_{0} \mathrm{CH}_{3}$ an $\mathrm{O}$ und $6 \cdot 15 \% \mathrm{CH}_{3}$ an $\mathrm{N}$; ber. für $\mathrm{C}_{19} \mathrm{H}_{16} \mathrm{O}_{2}\left(\mathrm{~N}\left(\mathrm{CH}_{3}\right)_{2}\right) \mathrm{J}$. $(\mathrm{OH})\left(\mathrm{OCH}_{3}\right)=\mathrm{C}_{22} \mathrm{H}_{26} \mathrm{NO}_{4} \mathrm{~J} 3.03 \% \mathrm{CH}_{3}$ an $\mathrm{O}$ und $6.07 \% \mathrm{CH}_{3}$ an $\mathrm{N}$.

$\mathrm{Ob}$ auch hier Aufspaltung der Oxydbindungen und Anlagerung von $\mathrm{H}_{2} \mathrm{O}$ eingetreten ist, ließe sich nur durch eine Verbrennung entscheiden, die aber bei diesem weit zurückliegenden Versuch nicht gemacht wurde. 
$B$. Die Methylierung des $\beta$-Bebirins nach Pschorr ${ }^{1}$ wurde mit $3 g$-Bebirin vorgenommen, wobei die Mengenverhältnisse genau wie bei der Methylierung des Morphins eingehalten wurden. Das durch Fällen mit konzentrierter JK-Lösung erhaltene Jodid wurde nach dem Umkrystallisieren aus Wasser, in dem es ziemlich schwer löslich ist, analysiert. Sein Schmelzpunkt liegt bei raschem Erhitzen bei $244^{\circ}$ unter Zersetzung.

I. $0 \cdot 2121 g$ Substanz (bei $100^{\circ}$ getrocknet) gaben $0.3892 g \mathrm{CO}_{2}$ und $0.1021 \mathrm{~g} \mathrm{H}_{2} \mathrm{O}$; II. $0.2371 \mathrm{~g}$ Substanz (bei $130^{\circ}$ getrocknet) gaben $0.4324 \mathrm{~g}$ CO. und $0 \cdot 1158 g \mathrm{H}_{2} \mathrm{O}$; III. $0 \cdot 2795 \mathrm{~g}$ Substan $z$ (bei $100^{\circ}$ getrocknet) gaben $0 \cdot 2674 \sigma$ $\mathrm{JAg}$ bei der Methoxyl- und $0.1080 \mathrm{~g} \mathrm{JAg}$ bei der Methylimidbestimmung (Wiederholung derselben negativ); IV. $0.0605 \mathrm{~g}$ Substanz (bei $130^{\circ}$ getrocknet) gaben nach Zerewitin of bei $b=737 \mathrm{~mm}$ und $t=22^{\circ} 10 \cdot 80 \mathrm{~cm}^{3} \mathrm{CH}_{4}$.

Gef.: I. $50 \cdot 05 \%$ C, $5 \cdot 35 \%$ H; II. $49 \cdot 74 \%$ C, $5 \cdot 43 \%$ H; III. $6 \cdot 10 \%_{0}$ $\mathrm{CH}_{3}$ an $\mathrm{O}$ und $2 \cdot 57 \%_{10} \mathrm{CH}_{3}$ an N; IV. $11.98 \%$ OH $(3 \cdot 74$ Hydroxylzahl statt 4$)$. Ber. für $\mathrm{C}_{19} \mathrm{H}_{17}\left(\mathrm{NCH}_{3} . \mathrm{J}\right)(\mathrm{OH})_{4}\left(\mathrm{OCH}_{3}\right)_{2}=\mathrm{C}_{22} \mathrm{H}_{30} \mathrm{NO}_{6} \mathrm{~J} 49 \cdot 720_{10} \mathrm{C}, 5 \cdot 690_{0} \mathrm{H}$, $5 \cdot 65 \% \mathrm{CH}_{3}$ an $\mathrm{O}, 2 \cdot 82 \% \mathrm{CH}_{3}$ an $\mathrm{N}, 12 \cdot 81 \%$

$1 \mathrm{~g}$ des Jodids wurde 20 Minuten lang am Wasserbad mit konzentrierter $\mathrm{H}_{2} \mathrm{SO}_{4}$ erwärmt. Nach Verdünnen mit Wasser und Filtrieren des ausgeschiedenen Jods und verkohlter Teile wurde mit konzentrierter JK-Lösung ein Jodid gefällt, das sich, wie die Verbrennung zeigt, aus dem Ausgangskörper durch Verlust von $1 \mathrm{Mol}_{2} \mathrm{O}$ gebildet hat:

$0.1954 \mathrm{~g}$ Substanz (bei $100^{\circ}$ getrocknet) gaben $0.3709 \mathrm{~g} \mathrm{CO}_{2}$ und $0 \cdot 0967 g \mathrm{H}_{2} \mathrm{O}$.

Gef. $51 \cdot 770 / 0 \mathrm{C}, 5 \cdot 50 \% \mathrm{H}$; ber. für $\mathrm{C}_{22} \mathrm{H}_{28} \mathrm{NO}_{5} \mathrm{~J} 51 \cdot 45 \%_{0} \mathrm{C}, 5 \cdot 500 \% \mathrm{H}$.

\section{Das inaktive Umwandlungsprodukt des $\beta$-Bebirins.}

Als eine größere Menge von $\beta$-Bebirin zur Reinigung nochmals in Benzol gelöst werden sollte und zu diesem Zwecke am Rückflußkühler längere Zeit damit gekocht wurde, zeigte es sich, daß fast nichts in Lösung gegangen war. Nach dem Abdestillieren des Benzols blieb nur ein kleiner Rückstand. Das so veränderte Alkaloid erwies sich als in Alkohol ziemlich (wenn auch bedeutend schwerer als $\beta$-Bebirin), in Pyridin sehr leicht löslich. Als das optische Drehvermögen der neuen

1 Ber. Deutsch. chem. Ges., 44, 2633 (1911). 
Substanz in Pyridinlösung bestimmt wurde, erwies sie sich als optisch inaktiv. Die Ursache dieser Veränderung lag jedenfalls an einer unbekannten Beimengung des verwendeten Benzols, die diese Verwandlung katalytisch beschleunigte. Denn der Rest des $\beta$-Bebirins löste sich in Benzol, das einer neuen Füllung entnommen wurde, leicht auf, war also unverändert. Oftmals noch versuchte ich diese Veränderung wieder $\mathrm{zu}$ erreichen, doch immer ohne Erfolg. Dieser inaktive Abkömmling des $\beta$-Bebirins zeigte außer seiner Schwerlöslichkeit noch andere abweichende Eigenschaften, die darauf hinwiesen, daß nicht etwa bloß die Racemform des $\beta$-Bebirins vorlag. Wenn er mit verdünnter Säure erwärmt wurde, um als Salz in Lösung gebracht zu werden, so war die Fällung mit $\mathrm{Na}_{2} \mathrm{CO}_{3}$ aus der Salzlösung im Gegensatz zum Bebirin nie eine vollständige. Mit $\mathrm{J}_{2}$.JK-Lösung wurde im Filtrat immer eine beträchtliche Fällung erreicht. Dies ließ vermuten, daß in saurer Lösung zum Teile ein Übergang in eine quartäre Verbindung stattfindet. Die späteren Beobachtungen bestätigten dies. In Eisessig war die inaktive Base unlöslich, während Bebirin darin leicht sich löst. Auf Zusatz von $\mathrm{H}_{2} \mathrm{O}$ trat aber sofort Lösung ein.

$\mathrm{Daß}$ die inaktive Base noch tertiärer Natur war, ergab sich daraus, daß sie mit Methylalkohol und 3 Molen $\mathrm{JCH}_{3}$, im Bombenrohr $2 \frac{1}{2}$ Stunden auf $100^{\circ}$ erhitzt, ein Jodmethylat lieferte mit dem Zersetzungspunkt $245^{\circ}$ bei raschem Erhitzen. ${ }^{1}$

I. $0.2198 \mathrm{~g}$ der ungereinigten; bei $100^{\circ}$ getrockneten Substanz gaben nach Carius $0 \cdot 1165 \mathrm{~g} \mathrm{JAg}$; II. $0 \cdot 2413 \mathrm{~g}$ Substanz gaben $0 \cdot 1194 \mathrm{~g}$ JAg bei der Methoxyl- und $0 \cdot 2364 \mathrm{~g} \mathrm{JAg}$ bei der Methylimidbestimmung.

Gef. $28 \cdot 65 \%$ J, $3 \cdot 16 \% \mathrm{CH}_{3}$ an $\mathrm{O}$ und $6 \cdot 25 \% \mathrm{CH}_{3}$ an $\mathrm{N}$.; ber. für $\mathrm{C}_{42} \mathrm{H}_{44} \mathrm{~N}_{2} \mathrm{O}_{7}\left(\mathrm{JCH}_{3}\right)_{2}=\mathrm{C}_{44} \mathrm{H}_{50} \mathrm{~N}_{2} \mathrm{O}_{7} \mathrm{~J}_{2}$ (siehe später) $26 \cdot 11 \% / 0 \mathrm{~J}, 3.09 \% \mathrm{CH}_{3}$ an $\mathrm{O}, 6 \cdot 18 \% \mathrm{CH}_{3}$ an $\mathrm{N}$.

Leider wurde die inaktive Base als solche nicht analysiert. Denn es wurden einige Zeit hindurch andere Arbeiten ausgeführt, die zur Folge hatten, daß die Luft des Zimmers oft mit feuchtem JH-Dampf erfüllt war. Als ich dann die Base wieder vornahm,

1 In der wässerigen Lösung ließ sich das $\mathrm{J}$ nicht durch Fällen mit $\mathrm{Ag} \mathrm{NO}$ nachweisen (erst durch Chlorwasser und Chloroform nachweisbar); ähnlich verhält sich das $\mathrm{Cl}$ im quartären Berberinchlorid. 
war sie zum größten Teile in das quartäre Jodid übergegangen. (Sie war durch den JH-Dampf viel stärker angegriffen worden als das $\beta$-Bebirin, das unter den gleichen Umständen nur zum kleineren Teile in das Jodid übergeführt wurde, jedenfalls infolge ihres Überganges in die stärkere basische quartäre Form.) Das Produkt war jetzt in siedendem Wasser vollständig löslich. Beim Erkalten fiel ungefähr die Hälfte als gelbe Flocken aus, die, wie die Analysen zeigten, größteils Jodid vermengt mit freier Base waren.

I. $0 \cdot 2280 g$ Substanz gaben $0 \cdot 4552 g \mathrm{CO}_{2}$ und $0 \cdot 1103 g \mathrm{H}_{2} \mathrm{O}$; Il. $0 \cdot 2129 g$ Substanz gaben $0.4305 \mathrm{~g} \mathrm{CO}_{2}$ und $0.0945 \mathrm{~g} \mathrm{H}_{2} \mathrm{O}$; III. $0.3067 \mathrm{~g}$ Substanz gaben nach Carius $0 \cdot 1236 \mathrm{~g} \mathrm{JAg}$.

Gef. : I. $54 \cdot 45 \%$ C, $5 \cdot 37 \%$ H; II. $55 \cdot 14 \%$ C, $4 \cdot 93 \%_{10}$ H; III. $21 \cdot 78 \%$ J.

Der in $\mathrm{H}_{2} \mathrm{O}$ gelöst bleibende Anteil, also jedenfalls Salz, wurde mit konzentrierter JK-Lösung als Jodid gefällt, ein weißer, breiiger Niederschlag, der nach dem Umkrystallisieren aus Wasser als gelbliches, unter dem Polarisationsmikroskop undeutlich krystallinisches Pulver erhalten wurde. Das Jodid erweist sich als quartär, da aus der wässerigen Lösung die Base weder mit $\mathrm{Na}_{2} \mathrm{CO}_{3}$ oder $\mathrm{NH}_{3}$ gefällt, noch aus der alkalischen Lösung mit $\mathrm{CHCl}_{3}$ ausgeschüttelt werden kann. Schmelzpunkt $250^{\circ}$ unter Zersetzung bei raschem Erhitzen. In $\mathrm{KOH}$ leicht löslich.

Wie die Verbrennungen des Jodids zeigen, muß die inaktive Modifikation des Bebirins durch Zusammentreten zweier Moleküle desselben unter Wasseraufnahme entstanden sein.

I. $0.2567 g$ bei $100^{\circ}$ getrockneter Substanz gaben $0.4827 g \mathrm{CO}_{2}$ und $0.1183 g \mathrm{H}_{2} \mathrm{O}$; II. $0.2874 \mathrm{~g}$ Substanz gaben $0.5441 \mathrm{~g} \mathrm{CO}_{2}$ und $0.1342 g \mathrm{H}_{2} \mathrm{O}$; III. $0 \cdot 2413 \mathrm{~g}$ Substanz gaben nach Carius $0.1226 \mathrm{~g} \mathrm{JAg}$; IV. $0.0921 \mathrm{~g}$ Substanz gaben nach Zerew itin off bei $b=746$ und $t=17^{\circ} 9.20 \mathrm{~cm}^{3} \mathrm{CH}_{4}$.

Gef.: I. $51 \cdot 29 \% / 0$ C, $5 \cdot 120 / 0 \mathrm{H}$; II. $51 \cdot 64 \% \%_{0} \mathrm{C}, 5 \cdot 19 \%_{0} \mathrm{H}$; III. $27 \cdot 45 \%_{0} \mathrm{~J}$; IV. $7 \cdot 05 \%$ OH. Ber. für $\mathrm{C}_{19} \mathrm{H}_{17} \mathrm{O}\left(\mathrm{NCH}_{3} . \mathrm{J}\right)(\mathrm{OH})_{2}\left(\mathrm{OCH}_{3}\right) \cdot \mathrm{O} \cdot \mathrm{C}_{19} \mathrm{H}_{17} \mathrm{O}\left(\mathrm{NCH}_{3} \mathrm{~J}\right)$. $.(\mathrm{OH})_{2}\left(\mathrm{OCH}_{3}\right)=\mathrm{C}_{42} \mathrm{H}_{50} \mathrm{~N}_{2} \mathrm{O}_{9} \mathrm{~J}_{2} 51 \cdot 420_{0} \mathrm{C}, 5 \cdot 14 \% \%_{0} \mathrm{H}, 25 \cdot 900_{0} \mathrm{~J}, 6 \cdot 940_{10} \mathrm{OH}$.

$\mathrm{Ob}$ die Aufnahme des Wassers unter Sprengung eines Sauerstoffringes schon beim Kochen in Benzol (vielleicht geliefert von dem vom amorphen Bebirin absorbierten Wasser, vgl. Gewichtsabnahme bei $100^{\circ}$ ) eintrat oder ob nicht vielmehr 
ein Zusammentreten zweier Mole Bebirin unter Wasserabspaltung vor sich ging und die Aufnahme von Wasser erst später unter dem Einfluß der feuchten JH-Dämpfe, beziehungsweise des Erhitzens in Wasser erfolgte, ist natürlich zweifelhaft. Die Inaktivität der Base wird dadurch wohl erklärt, daß bei der Bildung derselben die oxydartigen Bindungen des Bebirins, deren Haftstellen die drei asymmetrischen C-Atome sind, jedenfalls irgendwie verändert werden.

Auch das $\beta$-Bebirin selbst ging, wie erwähnt, beim Liegen an der oft mit Säuren gesättigten Zimmerluft in geringem Maße in in Benzol unlösliche Anteile über, die sich ebenfalls als stark jodidhaltig erwiesen. Durch Auskochen mit Wasser suchte ich die Salze möglichst zu entfernen. Der Rückstand, wohl zum größten Teil veränderte Base, zeigte einen sehr erhöhten Schmelzpunkt (unscharf bei $230^{\circ}$ ) und hohe Hydroxylzahlen, die auch wieder auf Aufsprengung der oxydartigen Bindungen schließen lassen:

I. $0.1267 \mathrm{~g}$ Substanz gaben bei $b=749$ und $t=22^{\circ} 21.6 \mathrm{~cm}^{3} \mathrm{CH}_{4}$, entsprechend $11.630 \% \mathrm{OH}$; II. $0.1152 \mathrm{~g}$ Substanz gaben bei $b=753$ und $t=22^{\circ} 19 \cdot 5 \mathrm{~cm}^{3} \mathrm{CH}_{4}$, entsprechend $11 \cdot 73 \% \mathrm{OH}$. 